\title{
Retrospective studies
}

\section{Les études rétrospectives}

n the present issue of The Canadian Respiratory
Journal, Bahadori et al (1) (pages e43-e49) exam-
ined the putative risk factors for the readmission(s)
of chronic obstructive pulmonary disease (COPD)
patients due to acute exacerbations. Using a stan-
dardized form, they examined the hospital charts of
patients admitted for acute exacerbations of COPD
over approximately 20 months in three Vancouver
(British Columbia) hospitals. They characterized
the patients and compared those who were subse-
quently readmitted with those who were not. The
paper exemplifies the strengths and weaknesses of
such an approach. An obvious problem resided in study timing. A patient's initial hospitalization occurred during the study period, regardless of whether he or she was previously hospitalized. Readmission had to occur in the same 20-month period to be counted; consequently, the risk of this event was necessarily related to how early in the study period the first exacerbation occurred; the longer a patient was eligible for a readmission, the more likely one was to occur. This may have been important in influencing readmission statistics.

A strength of the retrospective review is that it can accumulate data for a large number of patients, as this one did. It is also a strength that patients are unselected (ie, this is the 'real world'). Examining exacerbation rates in an ongoing clinical COPD trial has advantages in that patients are more likely to be standardized and more uniformly characterized, but they are, by definition, a subset of the 'real world'. A major weakness of retrospective studies is that they generate a great deal of missed data. For example, all would agree that the forced expiratory volume in $1 \mathrm{~s}$ is an important characteristic of patients with COPD, but it is very common - even in good hospitals - not to take this measurement during exacerbations. The same could be said for body mass index. Scrutiny of Table 1 of the Bahadori et al (1) paper shows that missing data were common in areas the authors believed were important. Missing data have the effect of reducing the size and power of a study, and if the missing data are not distributed randomly, are likely to inflict further damage. I believe, largely because of missing data, that significant risk factors for readmission were somewhat disappointing, amounting to previous home oxygen use and previous 'other respiratory disease'.

There were, however, some findings of real interest. There were distinct differences among the three hospitals studied regarding patient clientele, readmission rates and medication use. One of the hospitals clearly admitted patients that were less sick than the other two and, not surprisingly, had relatively low readmission rates. It also used antibiotics more frequently than the other two. The hospital with the highest readmission rates and arguably the sickest patients, also used less systemic steroid than the other two, which seemed bizarre. Indeed, if the ans le présent numéro de la Revue canadienne de pneumologie, Bahadori et coll. (1) (pages e43-e49) se penchent sur les facteurs de risque potentiels de réhospitalisation chez les patients souffrant de maladie pulmonaire obstructive chronique (MPOC) due à des poussées aiguës de la maladie. Au moyen d'un formulaire standardisé, ils ont analysé les dossiers hospitaliers des patients admis pour poussée aiguë de MPOC au cours d'une période d'environ 20 mois dans trois hôpitaux de Vancouver (en Colombie-Britannique). Ils ont caractérisé les patients et comparé ceux qui avaient été réadmis à ceux qui ne l'avaient pas été. L'article met en lumière les forces et les faiblesses d'une telle approche. Un des problèmes évidents avait trait à la chronologie des événements au cours de l'étude. L'hospitalisation initiale d'un patient survenait durant la période de l'étude, peu importe qu'il s'agisse ou non d'une réhospitalisation. L'admission subséquente devait survenir au cours de la même période de 20 mois pour être comptabilisée. Par conséquent, le risque de réhospitalisation était nécessairement lié au délai entre le début de l'étude et l'exacerbation initiale. Plus il restait de temps au patient pour être réadmis à l'intérieur de cette période, plus la réadmission devenait probable. Cette situation a pu exercer une nette influence sur les statistiques de réadmission.

L'un des avantages des analyses rétrospectives comme celle-ci, est qu'elles permettent d'accumuler des données concernant de forts contingents de patients. Il est également intéressant que les patients n'aient pas été sélectionnés (c.-à-d., qu'ils provenaient de la population générale). L'analyse des taux de poussées dans une étude clinique continue sur la MPOC comporte des avantages puisque les patients sont plus susceptibles d'être standardisés et caractérisés de façon uniforme et qu'ils appartiennent par définition à une catégorie « de la population réelle ». En revanche, l'une des principales faiblesses des études rétrospectives est qu'elles sont propices à l'omission de beaucoup de données. Par exemple, tous s'entendraient pour dire que le volume expiratoire maximum en une seconde (VEMS) est un paramètre important chez les patients souffrant de MPOC, mais il est très fréquent, même dans les bons hôpitaux, de ne pas mesurer le VEMS lors des poussées de MPOC. Il en va de même pour l'indice de masse corporelle. Dans l'article de Bahadori et coll. (1), le Tableau 1 montre un manque flagrant de nombreuses données relatives à des domaines jugés importants par les auteurs. Ces données manquantes ont pour effet de réduire la taille et la puissance de l'étude et si elles ne sont pas distribuées aléatoirement, elles risquent d'être encore plus dommageables. Principalement à cause des données manquantes, j'ai été déçu par les principaux facteurs de risque de réadmission, soit antécédents d'oxygénothérapie à domicile et d'autres maladies respiratoires. 
patients' in-hospital medications were captured accurately, it could be argued that therapy was frequently suboptimal in three Vancouver teaching hospitals - a finding that should make us uncomfortable. Another somewhat ominous finding was that the risk of readmission was inversely related to the length of the original hospital stay. I would have expected the reverse, in that the sickest patients are usually the hardest to discharge, and this result suggests that at least some discharges were premature.

Nick R Anthonisen MD Editor-in-Chief, Canadian Respiratory Journal

\section{REFERENCE}

1. Bahadori K, FitzGerald JM, Levy RD, Fera T, Swiston J. Risk factors and outcomes associated with chronic obstructive pulmonary disease exacerbations requiring hospitalization. Can Respir J 2009;16:e43-e49.
Certaines conclusions revêtent toutefois un intérêt certain. On a noté des différences marquées entre les trois hôpitaux étudiés en ce qui a trait à la clientèle, aux taux de réadmission et à l'emploi des médicaments. L'un des hôpitaux admettait de toute évidence des patients moins malades que les deux autres. On ne sera donc pas surpris du taux relativement moindre de réadmission. Cet hôpital avait également recours aux antibiotiques plus souvent que les deux autres. L'hôpital qui présentait le taux de réadmission le plus élevé, et peut-être les patients les plus malades, avait également moins recours aux corticostéroïdes systémiques que les deux autres, ce qui a semblé bizarre. En effet, si la pharmacothérapie perhospitalière des patients a été fidèlement consignée, on pourrait dire que le traitement a souvent été sous-optimal dans ces trois hôpitaux universitaires de Vancouver, une conclusion dont il y a lieu de s'inquiéter. Autre observation plutôt inquiétante : le risque de réadmission était inversement proportionnel à la durée du séjour hospitalier initial. Je me serais attendu à l'inverse, c'està-dire qu'il est en général plus difficile d'accorder leur congé aux patients les plus malades. Or, ce résultat donne à penser que dans certains cas, les congés hospitaliers ont été accordés trop tôt.

Nick R. Anthonisen M.D. Rédacteur en chef, Revue canadienne de pneumologie.

\section{RÉFÉRENCE}

1. Bahadori K, FitzGerald JM, Levy RD, Fera T, Swiston J. Risk factors and outcomes associated with chronic obstructive pulmonary disease exacerbations requiring hospitalization. Can Respir J 2009;16:e43-e49. 


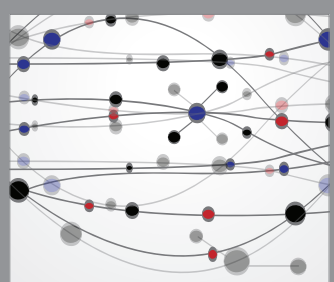

The Scientific World Journal
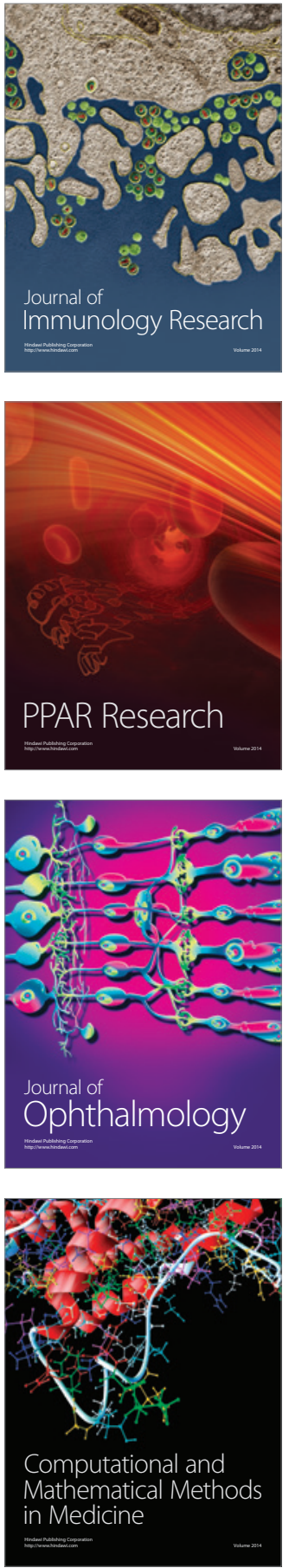

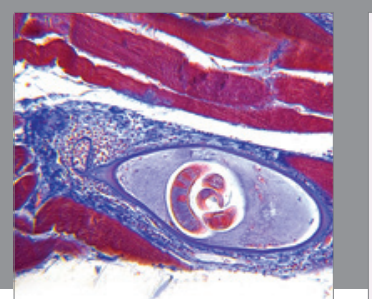

Gastroenterology Research and Practice

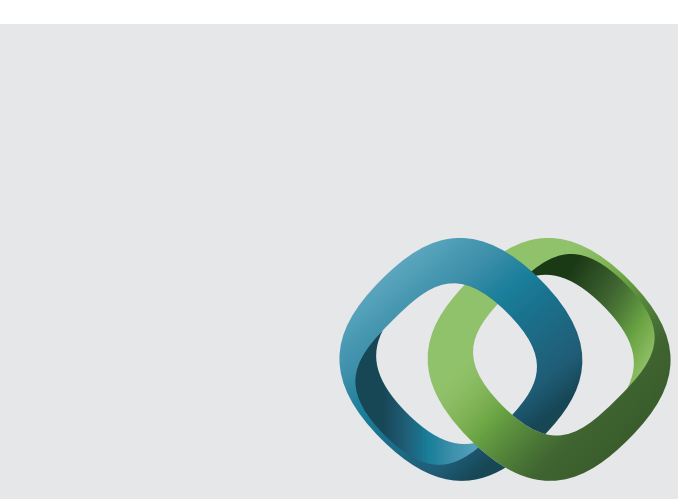

\section{Hindawi}

Submit your manuscripts at

http://www.hindawi.com
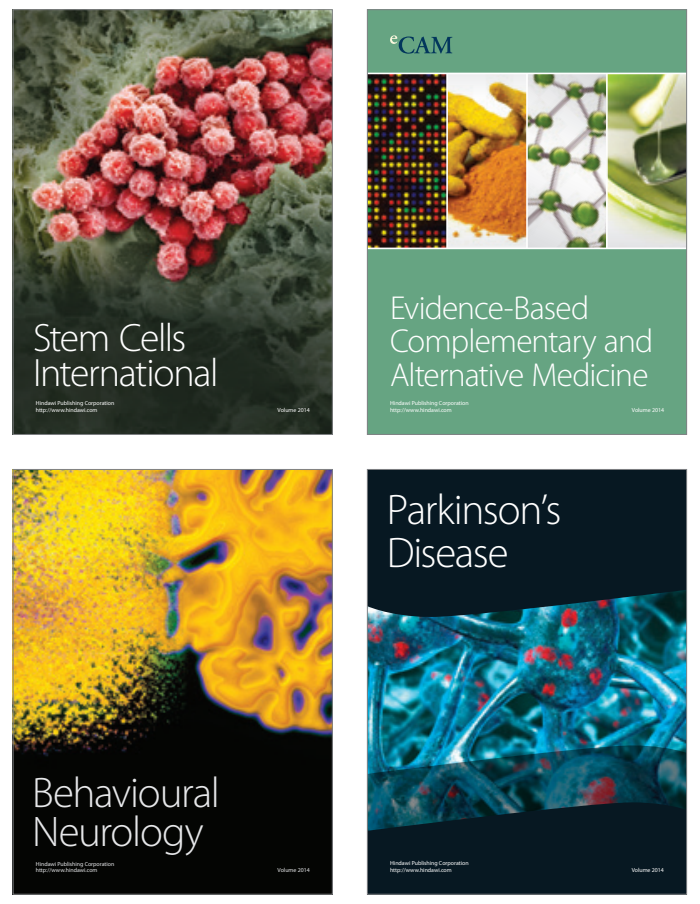
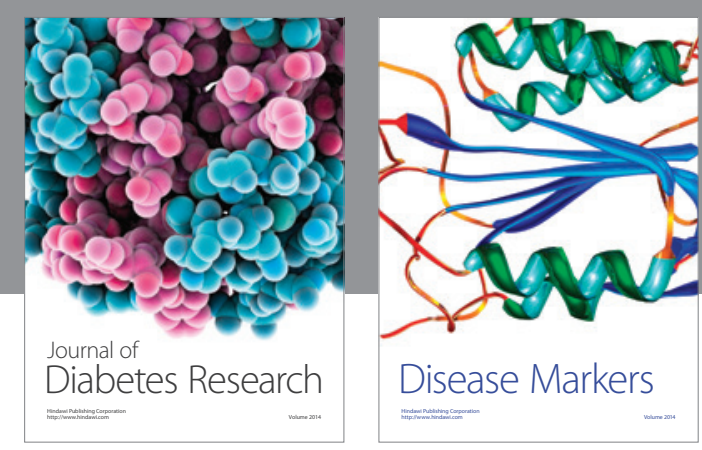

Disease Markers
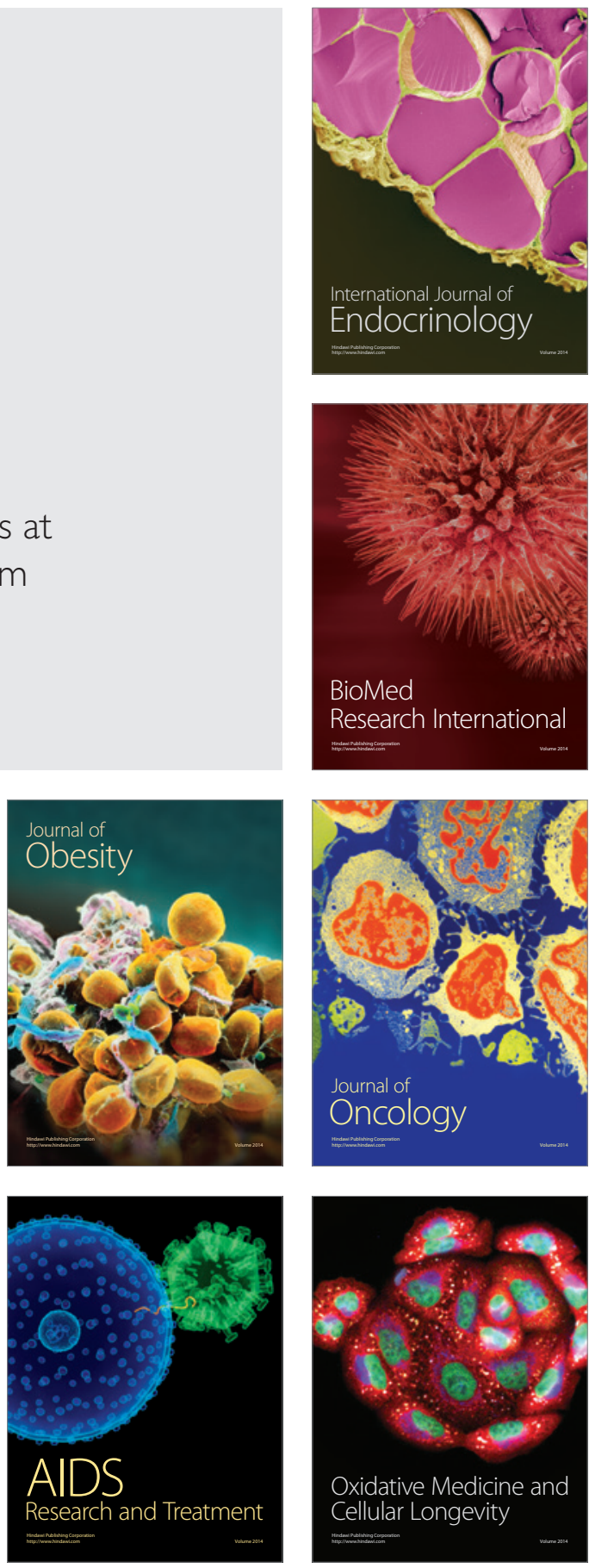